\title{
To change or not to change? Veterinarian and farmer perceptions of relational factors influencing the enactment of veterinary advice on dairy farms in the United Kingdom
}

\author{
Alison M. Bard, ${ }^{1 *}$ David Main, ${ }^{2}$ Emma Roe,${ }^{3}$ Anne Haase,${ }^{4}$ Helen Rebecca Whay, ${ }^{1}$ and Kristen K. Reyher ${ }^{1}$ \\ ${ }^{1}$ University of Bristol Veterinary School, Langford, Bristol BS40 5DU, United Kingdom \\ ${ }^{2}$ Royal Agricultural University, Cirencester, Gloucestershire GL7 6JS, United Kingdom \\ ${ }^{3}$ School of Geography and Environmental Sciences, University of Southampton, Southampton SO17 1BJ, United Kingdom \\ ${ }^{4}$ Cancer Prevention Program, Public Health Sciences Division, Fred Hutchinson Cancer Research Center, Seattle, WA 98109-1024
}

\section{ABSTRACT}

Achieving herd health and welfare improvement increasingly relies on cattle veterinarians to train and advise farmers, placing veterinary interactions at the heart of knowledge exchange. Cattle veterinarians recognize their influence and the need to be proactive advisors but struggle with acting upon this awareness in daily practice, reporting a need to enhance their advisory approach to inspire farmer behavior change. Understanding how veterinarian-farmer interactions positively or negatively influence the enactment of change on farm is therefore essential to support the cattle veterinary profession. This paper adopts a qualitative approach to conceptualize how and under what circumstances veterinary advice has the potential to support and inspire farmer engagement with behavior change on the UK dairy farm. Fourteen UK dairy farms were recruited to take part in a qualitative study involving research observation of a typical advisory consultation between veterinarian and farmer $(\mathrm{n}=14)$ followed by separate, in-depth interviews with the farmer(s) and their respective veterinarian. Interview data were organized using a template coding method and analyzed thematically. While accuracy of veterinary advisory content was valued, it was a relational context of trust, shared veterinarian-farmer understanding, and meaningful interpretation of advice at a local (farmer) level that was most likely to enact change. Critically, these relational factors were reported to work together synergistically; a trusting relationship was an essential, but not necessarily sufficient, component to create a culture of change. Findings suggest that cattle veterinarians may benefit from tailoring advisory services to the farmer's specific world view, facilitated by a shared understanding of the

Received January 24, 2019.

Accepted June 23, 2019.

*Corresponding author: alison.bard@bristol.ac.uk farmer's immediate and long-term motivational drivers. In consequence, cattle veterinarians seeking to positively engage farmers in advisory interactions could consider a focus on farmer priorities, motivations, and goals as paramount to frame and inform advisory messages. This explicit collaborative communication encourages the selection of appropriate and timely veterinary expertise, leading to better integration and adoption of advice on farm given enhanced advisory relevance for farmers' unique circumstances. This farmer-centered approach, involving active co-creation of plans between individuals, is critical for engagement and commitment when tackling complex problems.

Key words: veterinary advice, behavior change, dairy farmer, herd health, communication

\section{INTRODUCTION}

Achieving herd health and welfare improvement increasingly relies on cattle veterinarians (hereafter "veterinarians") to train and advise farmers (DEFRA, 2004; FAWC, 2011), placing veterinary communication and advisory services at the forefront of herd health management. Veterinarians recognize their influence and the need to be proactive advisors but struggle with acting upon this awareness in daily practice (Cannas da Silva et al., 2006; Mee, 2007). In recent research, Ruston et al. (2016) identified that this struggle is so pervasive that veterinarians report challenges in influencing behavior change as fundamentally undermining the preventative advisory role itself. As one male partner in the Ruston et al. (2016) veterinarian interview cohort indicated, "I think the battleground is probably not on the science, the battleground is on behavior change and all this type of thing. So it's not knowing more stuff that we need - we need to basically be able to implement it better."

In the veterinary sciences, research efforts aiming to characterize the intricacies of farmer behavior have 
been dominated by the adoption of theoretical frameworks from psychological sciences, most notably the theory of planned behavior and the health belief model (Ritter et al., 2017). This has generated a plethora of studies in the behavioral approach (Ritter et al., 2017) seeking to understand individual decision maker behavior, focusing on psychological constructs such as goals, attitudes, and values and employing largely quantitative methodologies (Burton, 2004a). Recent publications placing increased emphasis on the sociological, rather than behavioral, perspectives have offered some insight into the herd health advisory paradigm, indicating various human factors implicit in the enactment of advice. For example, veterinarians report farmers' trust in veterinary knowledge and communication skills as important for implementation (Jansen, 2010), whereas the perceived role of the veterinarian, the relationship between veterinarian and farmer, and the trust invested in this relationship combine to effect adoption of advice (Richens et al., 2016). However, existing qualitative research tends to be driven by a specific disease or intervention focus, such as mastitis (Jansen, 2010) or vaccination (Richens et al., 2016), with no qualitative literature examining the veterinary advisory paradigm in and of itself.

This deficit in understanding means that there is little insight for veterinarians to use to support their services and promote more positive herd health discussions, nor theoretical basis for educators and trainers to tailor education packages to the specific needs and intricacies of this context. The aim of this study was to begin to address this knowledge gap and investigate veterinarian and farmer perceptions relating to the enactment of veterinary advice on UK dairy farms using a qualitative methodology.

\section{MATERIALS AND METHODS}

\section{Participant Recruitment and Sample}

Participating farmers were recruited through a multinational producer of dairy products. A regional operator approached all farms in a regional farmer group $(\mathrm{n}=$ 33) with information on the research study, from which a subset of farms $(\mathrm{n}=22)$ agreed to be contacted for recruitment purposes. Following contact by the main author (Bard), a final study sample of 14 farms ( $\mathrm{n}=$ 14) resulted where both the farmer(s) and veterinarian $(\mathrm{n}=11)$ were able to participate (some veterinarians were responsible for $>1$ farm in the sample). During the research process (Figure 1), a selection of farms opted to have multiple farm members attend the interview, meaning 19 farmers were interviewed across 14 farms. Additionally, one veterinarian became unavailable for

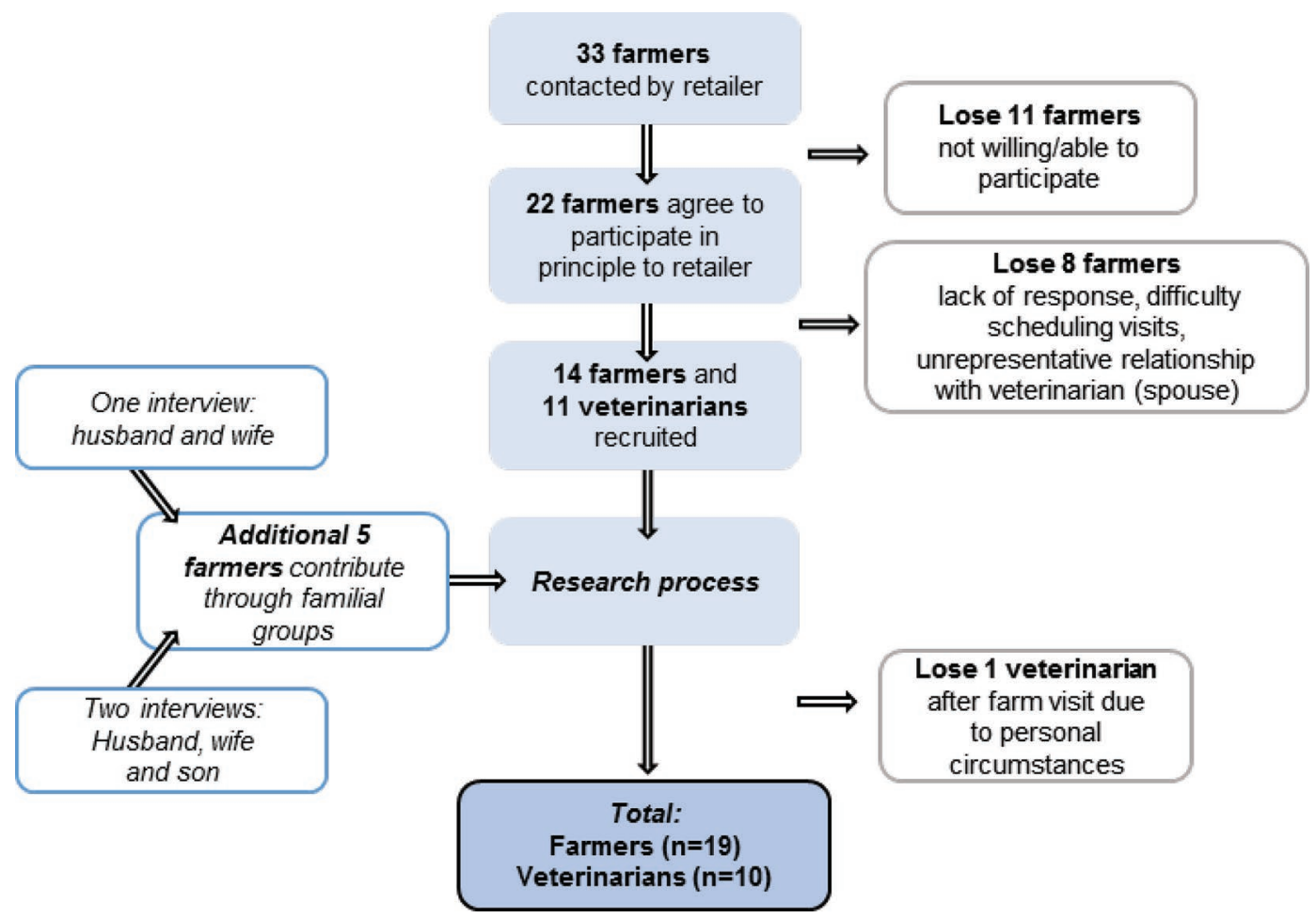

Figure 1. Flow diagram of recruitment process for total farmers $(\mathrm{n}=19)$ and veterinarians $(\mathrm{n}=10)$ across 14 farms. 
interview after the on-farm visit for personal reasons, resulting in 10 veterinarian interviews.

\section{Procedure and Data Collection}

The research methodology for each farm involved 2 distinct stages: $(1)$ research observation $(n=14)$ of a typical UK advisory consultation (i.e., a routine fertility visit, carried out at regular weekly to monthly intervals on the majority of year-round calving dairy farms) between veterinarian and farmer(s) followed by (2) an in-depth interview with the farmer(s) and an in-depth interview with their respective veterinarian $(\mathrm{n}=24)$.

(1) Each participating farm was visited by the main author (Bard) who was present during a routine veterinary consultation on cattle fertility involving the farmer(s) and their named veterinarian. This visit was an opportunity to observe and record by dictation machine a typical UK consultation between the veterinarian and farmer and gain an understanding of the complexities and contributing factors that shaped this interaction, for example, through observing the farm (layout, structure, handling systems, condition), the herd (herd size, behavior, condition), the farm staff (size, relationships to farmer, involvement, interactions), and the veterinarian-farmer interaction (familiarity, topics discussed, perceived habits, or routines).

Observations lasted a mean of 75 min (range 43 to 142 ) and provided the main author (Bard) with numerous insights that gave specific examples to discuss in the interviews that followed. While each interview developed along the same generic themes of the dynamic of interaction between veterinarian and farmer, expected and performed roles, and on-farm advisory behaviors, engaging the interviewee in specific grounded discussion about what happened during the observation enriched what could otherwise have been an abstract discussion. Brief field notes and salient photos were taken in the field to aid the analysis process of what was observed.

(2) Each party took part in an in-depth interview, conducted by the main author (Bard) and recorded by a dictation machine. Each semi-structured interview lasted a mean of 54 min (range 15-105 min) with the focus on eliciting decisions, processes, and perceptions relating to farmer behavior change in the context of advisory services. Interviews were iterative in nature, resulting in the foci of the interview schedule altering as the researcher's experience and insight into the topic area deepened; this allowed the main author (Bard) to more accurately follow the interviewees' interest, knowledge, and insights related to this topic (per DiCiccoBloom and Crabtree, 2006). For example, initial pilot questions used 2 endemic diseases (lameness and mastitis) as subjects through which to explore the enactment of veterinary advice on farm, given their resonance as topics of behavior change in the herd health advisory paradigm (Bard, 2018). However, through the interview process, it quickly became apparent to the main author (Bard) that inviting interviewees to recount their experience on the process of the delivery or receipt of advice on (1) behavior change topics of their choice, (2) behavior change topics observed during the farm visit, or both, provided more rigorous and detailed personal reconstruction of events and experiences, enhancing the experiential interview accounts and ensuring questions evolved responsively within each interview.

The pilot of this method was carried out on 2 farms and involved completing both interviews on farm following the herd health consultation. This approach was altered thereafter for all further interviews to secure separate interview locations for the farmer(s) and veterinarian to both remove any time pressure on the veterinarians and to create more perceived privacy for each interviewee's experience. Farmers were interviewed on-site after the observed consultation, and veterinarians were interviewed at their practice within $2 \mathrm{wk}$ of the visit.

Of the 2 pilot farms, the first set of interviews (veterinarian and farmer) was included in the analysis in their original form, whereas the second pilot farm participants (veterinarian and farmer) agreed to be re-interviewed 3 mo following the initial farm visit to spend more time on the in-depth interview process [the main author (Bard) re-visited the audio recording and notes of the farm visit in advance of these interviews]. All visits and interviews were carried out between March and June 2015.

An information sheet was supplied to participants detailing the aims of research before data collection, with written consent to take part obtained. This study was reviewed and approved by the University of Bristol Research Ethics Committee (ref. 14261), ensuring procedures met ethical guidelines in place for research with human participants.

\section{Interview Analysis}

Twenty-four interviews were transcribed (intelligent verbatim) by external transcribers for analysis. Transcripts and audio of a subset $(25 \%)$ of the interviews were initially explored using traditional paper-based coding methods, allowing assessment of the data and the development of initial coding ideas. Informed by this exploration, data were imported into the qualitative software NVivo 10 (QSR International, 2018) and organized/coded using the template methodology described by King (2004) to enable the comparison of farmer and veterinarian perspectives within this 
context. This coding process was inductive, with the template coding and structure determined and shaped by the data throughout the coding process. Once the full data set was coded, matrices were exported and analyzed thematically (Braun and Clarke, 2006), seeking to shed light on perceptions of why, and under what circumstances, advisory communication leads to the enactment of change for this sample of UK dairy farmers and cattle veterinarians.

\section{Research Team}

Analysis was carried out by the main author (Bard). Coded transcripts and thematic content were shared and discussed throughout the main author's $\mathrm{PhD}$ studies (2014-2017) at regular meetings with all authors. These data were subsequently cross-examined by one female supervisor (Roe, an experienced social and cultural geographer) during a lengthy assessment and conceptualization of the work immediately preceding the creation of this paper (August 2017) for submission within the main author's PhD thesis (April 2018).

\section{RESULTS}

\section{Participant Demographics}

Farmers in this study $(\mathrm{n}=19)$ were an average age of $42 \mathrm{yr}$ old (range 18 to $59 \mathrm{yr}$ old) and had been in dairy production for an average of 23 yr (range 3 to 45 yr). Their herds ranged from 60 cattle to 470 cattle, and 3 of the 19 farmers were female. Veterinarians in this study were an average of 44 yr old (range 25 to 60 yr old) and had been in farm practice an average of 19 yr (range 1 to $35 \mathrm{yr}$ ). Two of the 10 veterinarians were female.

\section{Themes}

Veterinarians and farmers spoke about 3 core factors that influenced whether advice would be enacted on farm: the context-bound capacity for advice to manifest meaning, the belief in the virtue(s) in the veterinarian that lay the foundation for relational trust, and the foundation of a shared understanding between veterinarian and farmer.

\section{Meaning Is Manifest at a Local Level}

Veterinarian 9: "To be honest it is very complex, it really is. And there is no telling who is going to listen to your advice, and who isn't... I know the very narrow veterinary aspect, but there are so many factors in the game, from price of milk, to relationship with dad, to relationship with the bank manager, to you know."

Underpinning the multitude of descriptions on enacting change was one common narrative: that for knowledge to be enacted a farmer must interpret the advice as meaningful in the local context of their farming world view. However, as the veterinarian quoted above recognized, there are myriad "factors in the game" that contribute to this local interpretation, creating a complex web of interconnected considerations for the farmer that act in synergy to evaluate an advisory topic. Interview data suggest that for advice to manifest meaning in the farmer's eyes, it needed to either be congruent with the farmer's world view or, if it was not congruent, sufficiently salient to catalyze the recalibration of this world view in a way that would lead to integration.

Congruence with the World View. The world view of the farmer was invoked through the integration of diverse factors, broadly relating to the aspects of the farmer's individual, social, and environmental influences; those explored by interviewees are presented in Figure 2. With regard to aspects of social influence, narratives included the farmers' friends and family, onfarm hierarchies, the farming community, the veterinarian and their practice, other advisors (agronomists, foot trimmers, nutritionists, and so on), retailers, farm assurance, and the nonfarming public. For example, when one farmer discussed his approach to field management around his farm, the social effect that the farming community could have on his enacted behavior was clear:

Farmer 9: "I own that piece of land out on the dual carriageway as you turn in. It's right on the dual carriageway. Every farmer goes past that and it rises up from the road. I grow maize there. That field gets everything it needs because every farmer looks at that."

For this farmer, their world view might include the narrative "I want to be perceived as a good farmer." External recommendations pertaining to the flourishing of this field in view of the farming community would therefore be perceived as valuable, due to maintenance of social status [a phenomenon recognized by Burton (2004b) as part of "roadside farming," where perceived social significance and management behavior(s) interact].

The second area of influence was aspects broadly considered as environmental: farm factors (restrictions of system, tenancy structure, routines dictated by the farm physical set up), season, market, and milk buyer. For example, one farmer reflected on how their decision to put milking robots on their farm the year before 


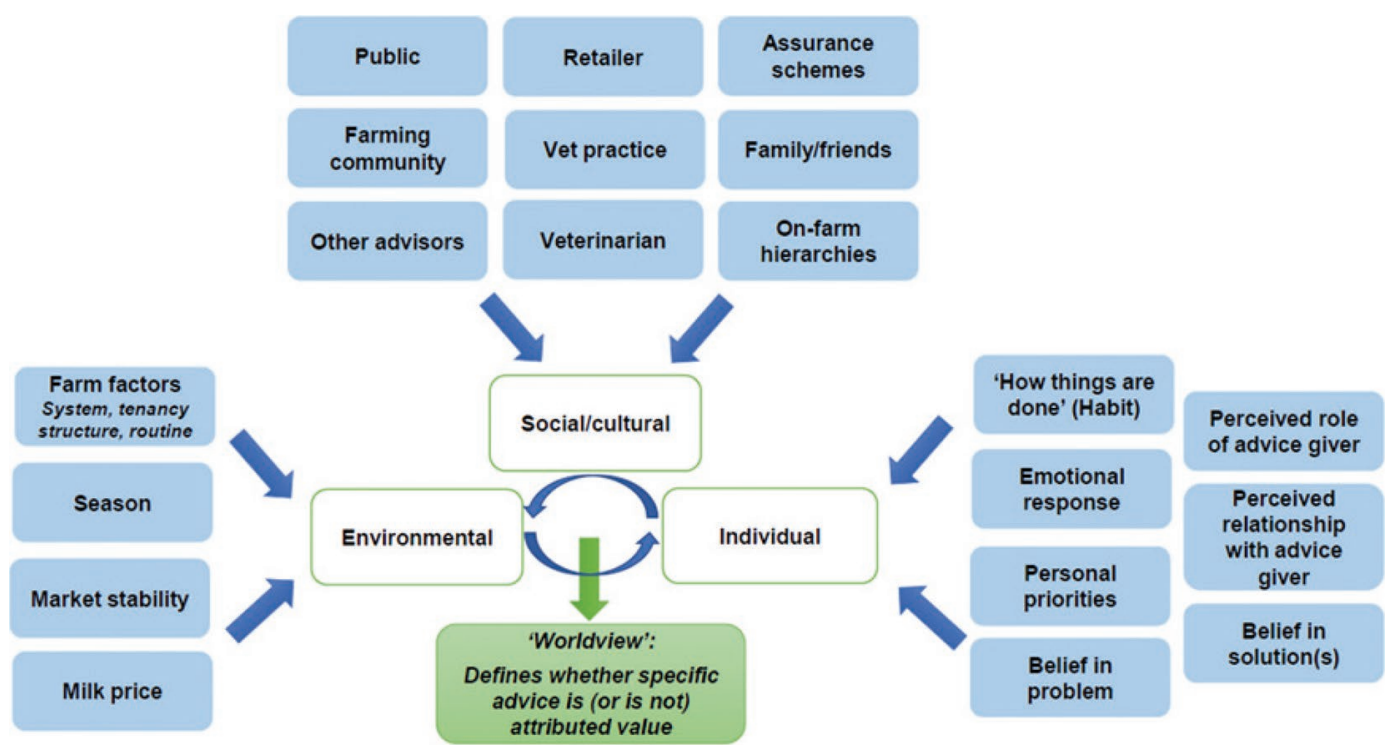

Figure 2. Factors reported by interviewees as contributing to the world view of the farmer, broadly relating to aspects of the farmer's individual, social, and environmental world.

would not have happened in the current climate, given the need to watch every cost and be cautious with expenditure:

Farmer 7: "I think, at the moment, what would hold people back is, dare I say it, milk price. Because it's restricting of, you know, farmers having to watch every cost... This time last year we were just started putting in our second robot. If it were the same time now, with our milk price, we wouldn't have done that."

The farmer's world view appears to have moved from something like "We can be optimistic and invest in the farm" to "It pays to be cautious at present," which they perceive as influential on how they interpret information and make decisions, so much so that the decision to enact a significant management change is seen completely differently when considering subsequent changes in the milk price.

The final area of influence can be considered as pertaining to the individual farmer: their priorities, belief in solution(s), belief in the problem, habitual processes, emotional responses, and perceived role of and relationship with the advisor. For example, farmer perception of the advisory role could influence engagement; one veterinarian reflected upon the difficulties of engaging farmers in proactive advisory interactions when their perceived role was more technical:

Veterinarian 8: "I asked him what his cost of production was a few months ago now and I think his response was "What do you think you are? A consultant"?... I feel like I just go and PD [pregnancy diagnose] his cows, which is kind of wrong, because he could turn around and get a technician, I guess, to do what I do. But I don't really advise him that much."

For the farmer, this interaction perhaps reflects a farming world view of something like "I need my veterinarian for practical fertility work, but for business matters I go elsewhere," meaning the veterinarian's advice would be unlikely to manifest meaning in the area of production costs.

These influences are not stand-alone aspects as the farming world view is a cumulative synergy. For example, if the above scenarios framed the internal narrative of one individual, their wish "to be perceived as a good farmer" would have to balance their sense that "it pays to be cautious at present." As such, the value of behavior that enables the field seen by the farming community to flourish may be diminished by the need to spend extra money when in a cautious mindset.

Catalysts for Recalibration of the World View. If a recommendation was not aligned with this existing world view, this did not (necessarily) mean it would not be enacted. Certain circumstantial aspects of advice giving could recalibrate farmer interpretation of advisory content, which can be broadly thought of as those relating to the practical or relational mode of advisory delivery.

(1) Relational Saliency. In this interaction, the veterinarian reflects on an instance when their advice 
spontaneously found meaning after $7 \mathrm{yr}$ of the same message:

Veterinarian 3: "A classic was I'd been working on one guy for about 7 years about his mastitis and how he milked his cows. He'd start at the front, going all the way down and wiping the cows. And then come all the way back, putting the clusters on. And I was trying to tell him, 'Go back to the front and do it the same way.' Then we had a mastitis meeting one evening and [respected industry specialist] said just the same thing, and he did it overnight... The guy started it the next day and never looked back."

For this farmer, the relational context under which the advice was given embedded the advice with new meaning. It was the advisor giving the message, more than the message itself, that gave the message saliency and inspired enactment.

This relational enactment of meaning was recognized in myriad circumstances, for example interactions between farmers and respected speakers (such as at group meetings with industry specialists, industry conferences, producer meetings), specialist advisors (nutritionists, foot trimmers, agronomists), other farmers, family, and friends.

(2) Delivery Saliency. Advisory meaning could also manifest because of delivery saliency. Novel messages where farmers were able to see the change in action, such as seeing another farmer or veterinarian using a new piece of equipment or viewing the results of change on other farms (whether in action or through improved health and production figures) enhanced the saliency of management recommendations:

Veterinarian 9: "That pump that [farmer X] was mentioning... 'No, we don't want to buy that, it cost $£ 80.00$ !'. . . Then something happened... he saw that when we drenched cows with our pump it worked. So he bought a pump. And all of a sudden that pump is fantastic."

Another aspect of delivery salience identified was the communication approach used by the veterinarian. Both veterinarians and farmers reported a variety of communication behaviors, attributes, and ethos (Appendix Table A1) that are desirable and undesirable in the dairy context. From both a veterinarian and farmer perspective, desired qualities tended to reflect a mutualistic communication paradigm, for example where client opinions were actively sought, negotiation and collaboration led to an openly agreed upon plan, and active empathic skills are used (Roter, 2000):
Farmer 1: "Vets do know the academic side. They're bright lads and lasses. But sometimes it doesn't hurt to stop talking, and start listening, when you go on farm."

Undesirable communication attributes were generally associated with making the farmer feel less than the veterinarian in some way, such as chastising, blaming, judging, using jargon, rudeness, or assuming farmer wants or needs:

Farmer 8: "I won't go back to those that think, 'I'm a professional. And you're just a dairy farmer."'

Both veterinarians and farmers reported desirable communication features as associated with positive outcomes, such as engaging farmers better in conversations, protecting a sense of pride, promoting ownership over behavior changes, and enhancing satisfaction and adherence to veterinary recommendations.

The means of delivery of advice, whether providing information in person, in paper form, via email, by phone, or by tablet, was felt to provide different opportunities for engagement and understanding. For example, one veterinarian reflected on his habit of following an advisory discussion on an National Milk Record (NMR, 2018) report by leaving a hard copy of the elements discussed with the farmer:

Veterinarian 3: "I tend to leave [the report hard copy] there so they can go back and think, 'Oh, what was he on about?' But also, it just lets it tickle in their mind. ... The best way of getting things to change is if they think about it, and want to change, rather than they feel they've got to because you've told them."

For this veterinarian, the integration of multiple delivery mechanisms allowed their advice to be present on the farm in their absence, moving it from something to be pushed on the farmer in the moment to something that could be mulled over and engaged with as a choice. Veterinarians reported working out by trial and error which farmers would be receptive to which delivery types to allow their advice to permeate beyond the boundaries of just face to face contact to enhance saliency.

Finally, novel messages that were felt to be consistent with those held by other social contacts, such as within veterinarians in the same practice, between veterinarians and outside advisors (such as foot trimmers), or between veterinarians and farming contacts, were reported to have the potential to be viewed more favorably. 


\section{The Belief in Virtue}

Farmer 12: "Oh God yes, yes, 100\%. It's got to be. It takes a long time to build that trust up and it's only done over time from seeing what [sick] animals recover from their examination [of the animal], from their points of view [as to] what's wrong. And yeah there has to be a lot of trust there, which is why I find it strange when people jump from one veterinary practice, to the next, to the next."

Throughout these interviews, veterinarians and farmers spoke at length about a critical bond of trust between them; their professional relationship was predicated upon this attribute. The importance of establishing this relational bond was witnessed in narratives on the working relationship, where virtues that secure trustworthiness (Figure 3) manifest in stories of what defines the ideal farm veterinary experience.

Ability. The perceived ability of the veterinarian was a critical foundation of the interaction, with both parties expressing a perceived correlation between the veterinarians' overall experience and this virtue. This overall experience captured traits of both scientific/ professional knowledge (age, length of time in practice, specialization, mixed/specialist practice) and local knowledge (personal background in or out of farming, degree of personal and professional involvement in dairy context) suggesting that while ability in this context is founded upon scientific prowess, the virtue also encompasses employing this knowledge appropriately given contextual understanding. The value of ability was such that farmers would actively engage with advice when this virtue was perceived in their veterinarian, as perceived ability ensured accurate, reliable, and relevant herd health recommendations:
Farmer 3: "Yes, we are lucky that [our veterinarian] is the best vet that is up there. He has got experience. And he does talks all over the world. And he is a pretty knowledgeable chap, so what he says you sort of listen to... His quality is his knowledge."

Veterinarians showed an awareness of this through their cultivation of ability signals, such as being a specialist in a particular area (for example, having publications on a particular topic), seeking further qualifications [for example, through the Royal College of Veterinary Surgeons Certificate(s) in Advanced Veterinary Practice (RCVS, 2018a) or Advanced Practitioner status (RCVS, 2018b)], or emphasizing the longevity and closeness between themselves and their farmers and having a shared understanding of their local world.

Benevolence. The perception of benevolence threaded through narratives on the working relationship, where farmers expressed a desire for the veterinarian to deliver a service on compassionate grounds, one that was not strictly constrained by veterinary protocol and did not exist only to create veterinary profit, but that respected and had compassion for the needs and goals of the farmer(s). Veterinarians, in turn, were acutely aware of this benevolent side to veterinary services, reporting at times altering or adjusting service expectations and delivery based on the individual constraints and desires of the farmer they were interacting with. For example, veterinarians reported avoiding situations where they would have to deliver criticism to their primary farm clients, choosing instead to bring in another individual at the practice rather than thwart the perception of being benevolent:

Veterinarian 9: "If I told them that they're doing rubbish work at certain things they might take

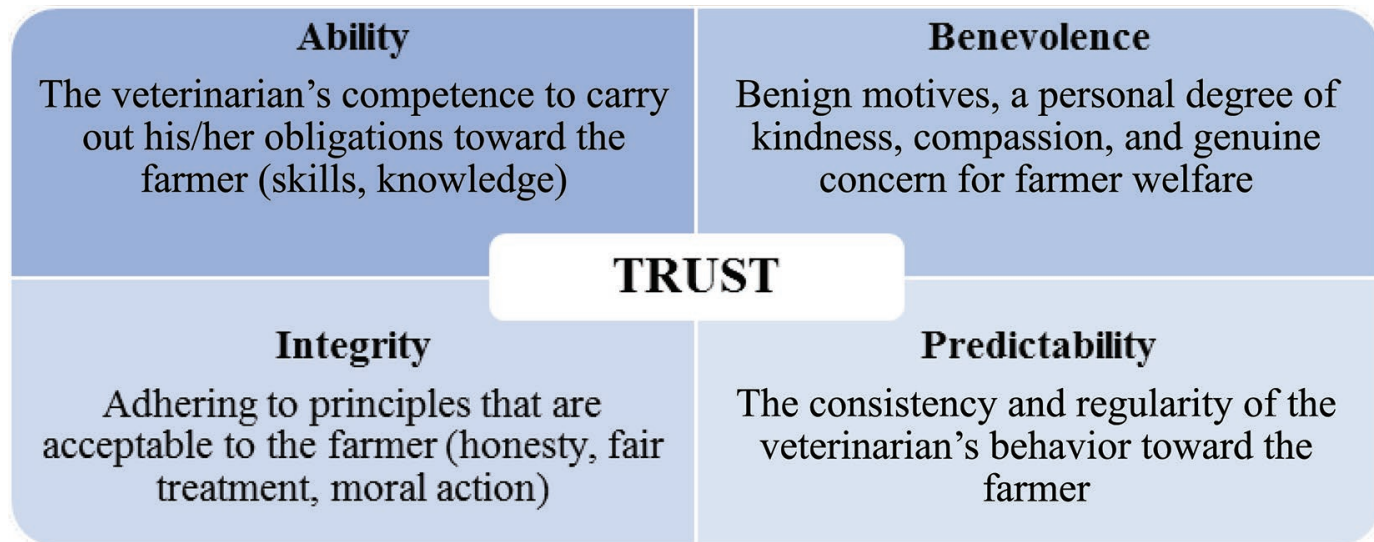

Figure 3. The 4 virtues needed for assessment of veterinarian trustworthiness (Dietz and Den Hartog, 2006). 
offence and that would impact on the relationship. Sometimes it is really nice to get somebody else on the farm, to tell them the bad things. And [then] you are still on good terms with them and you can then reemphasize."

Integrity. The need for integrity underpinned all aspects of the advisory interaction, where farmers' perceptions of this virtue instilled confidence in veterinary services. For example, farmers desired a sense that they received fair costings of treatment(s), the best advisory recommendations possible (in their unique circumstance), transparency on any mistakes made, and open acknowledgment of risks and "dead end" treatments:

Farmer 3: "You need someone honest as well, if someone says the cow is knackered, she is knackered, there is no point in trying. Whereas someone would say treat for this, treat for that. Sooner [I'd] have someone say 'She is knackered. It is not worth trying,' rather than spending money and having to shoot her later."

Veterinarians recognized the need for honesty to underpin their services with trust in their veterinary judgment sometimes stemming as much from honesty over things that they cannot do as much as ability in areas they have mastered:

Veterinarian 6: "Know what you can do. Know what you can't do. Be honest. If you do the things you say you can do, very well, and get someone else to help with the things you can't do, that instils a lot of confidence in them. They'll trust your judgment basically."

Predictability. Finally, veterinarian predictability encouraged a sense of security and stability in the advisory service. This sense of predictability arose through various factors, such as farmers having an individual they thought of as their veterinarian at a particular practice who was primarily responsible for their routine visits, having a veterinarian who could be relied upon to support them in emergencies (access to the veterinarian's mobile phone number was often mentioned as indicative of this support), and having someone who could be relied upon to be connected with them over the long term. One farmer's "twitchiness" at having to change veterinarians reflects this need for stability and predictability:

Farmer 10: "We've been with [veterinarian X] a long, long time now... oh, 10 or 15 years, I suppose... We had some other vets for a while in there. They weren't partners, they were just employed, and they kept leaving. ... I was getting a bit twitchy about it if I'm honest... This is not good. You just get into a routine with one vet, how they work, and they know how I work and they announce they're leaving. ... So it is quite nice to have that stability with [veterinarian $\mathrm{X}]$... I've got his mobile phone number if I need to ask him any questions."

The culmination of the virtues underpinning trustworthiness is well illustrated in this veterinarian's statement on the working relationship:

Veterinarian 9: "They trust you and they believe in you. And you are entrusted with something, as I said, quite sacred to my mind, because you mustn't bluff. You should try to do your best at all times. Even if you are tired, and completely broken and you have had three horrible nights of cold. If he then needs you... you can say, 'Alright, I will jump in the car.' And then I will go today."

In this one statement the veterinarian has echoed the need for ability ("doing your best at all times"), integrity ("don't bluff"), predictability ("even if you are tired and broken... you jump in the car"), and benevolence, where the overall description intuitively conveys an approach embedded in kindness and concern.

The sense of trust between farmer and veterinarian was reported to build up over time and become embedded through a variety of attributes of the working relationship (Table 1), facilitating the decision to trust and enactment of trust (advisory behavior change). This contextual development offers some benefits to veterinarians; both parties recognized the protective effect of trust between veterinarian and farmer. Once this trust was established, farmers would become more forgiving of mistakes given a strong perceptual establishment of these virtues (perhaps underpinning why both parties reported mistakes early in a veterinarian's relationship as particularly damaging).

It is important to note that trustworthiness was not necessarily perceived in an all or nothing manner but could be attributed by farmers in degrees, based on the management topic under consideration and how the farmer interpreted veterinarian trustworthiness in this area. For example, one farmer was happy to receive his veterinarian's advice on animal health but very reluctant to engage in any discussion of production costs.

Trust could also be ameliorated by the depth, strength, longevity, and loyalty of the relationship in question, varying from professional colleagues to personal friends from farm to farm. Interestingly, it was 
not that some veterinarians or farmers were particularly likely to be friends with their clients (or vice versa) but a synergistic effect of individual veterinarian-farmer dyads; one veterinarian could be close friends with some farmers and not others, whereas some farmers found their veterinary relationship shifted with engagement of a new veterinarian.

\section{A Shared Understanding}

Both veterinarians and farmers reported the need for a shared understanding with the farmer, of his or her world view, perspective, and myriad aspects that could act as barriers and motivators to enacting change. This shared understanding shaped veterinarian choices about advisory communication, farmers' proclivity to engage with advisory communication, and the consultation paradigm itself.

Veterinarian Advisory Choices. Veterinarians reported 2 levels to understanding the farmer: a need to understand the dairy farming context, combined with an understanding of the individual farmer and his/her farming world view (the way that they perceive the farming world in which they are situated):

Veterinarian 8: "I'd say try and get a really good understanding of how dairy farms run. And try and see as many farms as you can. And I think just treat each farm as an individual. Don't look at all farmers as the same, [be]cause some will, yeah, want to do things that others don't. ... Everybody has different aspirations."

Veterinarians often spoke about this shared understanding with pride, feeling that their in-depth knowledge offered them the chance to provide a unique and valuable service to their farmer(s) that is often qualitatively different from what can be provided to clients in small animal services. Indeed, veterinarians felt farmers recognized this as part of the added value in their service:
Veterinarian 2: "I think you understand their relationship and needs better when you've had that continual link. Somebody coming in [to small animal practice] you have to start again really to try and understand what they really want... we have all this intellectual property on their farms really."

This ability to connect with the localized reality of the farm could influence all aspects of advice giving on behalf of the veterinarian, from the topics broached and interpretation of diagnostic protocols taken, to the advice given and parameters set for success. Veterinarians reported an ability to make appropriate judgments and decisions on their advisory approach and recommendations made, through knowing whether topics would be likely to be received positively or negatively (and thus whether it is worth broaching the topic), what actions would be feasible for the farmer in question, and what type of delivery of advice the farmer would be most receptive to:

Veterinarian 10: "Because you know them well, you know what their expectations are likely to be. There are certain cases you would treat differently, on different farms."

Veterinarians would often use this insight on their farm clients to group them by the valence of their broad overall response to advisory recommendations. While varied in name, these group labels or farmer types were semantically similar and broadly reflected binary divisions of whether farm clients were likely to enact complex change (positive) or unlikely to enact complex change (negative): for example, proactive and reactive, good and bad, advice takers and advice leavers, motivated and unmotivated, and listeners and nonlisteners.

Veterinarian 9: "It is probably farmer's type. Some would listen to advice. And some won't listen to advice and crash and burn."

Table 1. Attributes of the veterinarian-dairy farmer working relationship offering the opportunity for the development of veterinarian trustworthy virtues

\begin{tabular}{ll}
\hline Attribute & Description \\
\hline $\begin{array}{l}\text { Longevity } \\
\text { Intensity }\end{array}$ & $\begin{array}{l}\text { Many veterinarian-farmer relationships are established over years or even decades. } \\
\text { Intense interactions are par for the course, such as working under stressful conditions late at night together } \\
\text { for long periods, or the veterinarian being there for the farmer in times of crisis on the farm. }\end{array}$ \\
$\begin{array}{l}\text { Frequency of communication } \\
\text { Sociality }\end{array}$ & $\begin{array}{l}\text { Mest herds will receive a routine consultation weekly or fortnightly to manage fertility, within which other } \\
\text { hommunity integration } \\
\text { The isolated nature of farming means veterinarians are often an important social contact for farmers. } \\
\text { The integration and involvement of both veterinarian and farmer in the wider farming/social community, } \\
\text { meaning shared personal contacts and overlapping social networks validate and strengthen the connection } \\
\text { between veterinarian and farmer. }\end{array}$ \\
\hline
\end{tabular}


This ability to categorize farmers illustrates how well veterinarians felt they shared an understanding of the farmer's context and world view. Through this categorization, veterinarians felt they were able to shape delivery of advice to maximize enactment on farm, making advice giving a situated activity; veterinary recommendations were an entanglement of scientific knowledge and local understanding.

Farmer Engagement. Farmers echoed veterinarian narratives on the shared understanding underpinning their advisory services. Many reported a desire to feel as if the veterinarian understood their unique farming context and farming world view, encouraging their veterinarian to act accordingly in the advisory process;

Farmer 15: "Yeah, and I think they need to understand what you want to do. And if you're [clear] they will. They'll know exactly what you want to do. And how focused you are to meet targets. And to get cows in calf. Or to achieve a growth rate. Or to fatten a store at a certain date or whatever. And I think they'll act accordingly."

This sense of being understood by the veterinarian could add meaning to the advice being conveyed, making it more salient through the perception of relevance to the individual farmer:

Farmer 11: "It's building up a relationship isn't it? ... Because I think my new vet's got more background knowledge [of my farm]. I would probably instigate any change on his doing, [more] so than I would have done in the past."

Farmers recognized that having a shared understanding shaped how veterinarians gave advice, with regard to the type of recommendations the veterinarian might make and their expectations of a farmer's response:

Farmer 8: "It's not necessarily knowing the farm as knowing the person. That personality you feel. That relationship... that's critical."

In this way, farmers also recognized advice giving was most valued as a situated activity, where veterinary recommendations could not be reduced to mere scientific knowledge; local understanding of the farmer, their context, and their farming world view were critical in meaningful delivery.

Consultation Paradigm. Interview and observational data suggest that this sense of a shared understanding was not just conceptual but was also manifested in the very behaviors surrounding the on-farm consultation paradigm, enacted between veterinarians and farmers in predictable and repeatable ways according to a socially perceived routine. This culturally shared expectation of events is well recognized and can be defined as a cultural script, a feature of social interactions of importance as scripts provide a framework for interaction (Vanclay and Enticott, 2011).

Within on-farm consultations, advisory communication was expected to informally pervade all points at which the veterinarian was present on farm:

(1) most typically, during (and often inextricable from) the practical obligations of cow- or herdspecific tasks (such as pregnancy diagnosis checks);

(2) permeating any point of the visit from the veterinarian exiting their vehicle at the beginning to climbing back in at the end (whether preparing equipment, cleaning boots, walking the farm, or drinking tea in the office);

(3) where paperwork or computer-based reports were necessary to oil the wheels of this communication, these were often informally presented within the farm environment rather than pursuing a more formalized sit-down meeting [e.g., Figure 4(a) and (b)];

(4) if a more formal sit-down interaction was to occur within a farm visit, the thread of informality would often be maintained by the location (the farm kitchen could be used), the continued integration of social and animal health communication, and the offer of hospitality (hot beverages, food, or both).

Additionally, socially oriented communication (friends, family, community, sport, leisure) was diffused throughout the consultation in the same way, making advisory communication mirror the process of a more personal engagement.

If veterinarians were not willing or able to adapt their advice to this informal consultation space, farmers would have to pay significantly more for their services, being charged for both the time spent in practical cowand herd-specific tasks in addition to a more formalized advisory consultation. While the latter certainly occur, the dominant paradigm was reported to be advice delivered informally during or bridging other tasks. This consultation paradigm, a cultural script of informality, therefore represents more than an ease of fit to the bounded environment of the farm consultations; it also implicitly signals that veterinarians share an understanding of the needs of the dairy farmer and prioritize a service that meets those needs, rather than focusing 
on maximizing veterinary profits by demanding structured advisory meetings separate from cow-side tasks.

\section{DISCUSSION}

\section{Interpreting This Study}

This research study took a qualitative approach to understanding nuance within the herd health advisory paradigm. This approach allows researchers to explore and uncover the complexity of interviewee experiences, rather than seeking to quantify opinions within a select group or generate a representative sample of those opinions (Vaarst et al., 2007). As such, the authors intend for the research findings to be ethnographically rigorous and valid in delivering detailed, context-specific insights on the veterinary advisory paradigm in action. The findings from this methodology could never claim to create a universal, representative picture of the paradigm in action, but importantly contribute understandings and nuance that positivist methodologies are ill-suited to grapple with.

It is important to consider the study sample may differ in meaningful ways from UK dairy farmers as a population:

(1) Engagement with the research was by choice, meaning study recruitment may have favored farmers with relatively better or more comfortable relationships with their herd veterinarian if this encouraged more favorable appraisal of the research topic.
(2) The cohort involved in this study - dairy farmers and their respective veterinarians in the southwest United Kingdom-may have focused research insight on factors that are linked in some way to this geographical context.

These factors may have introduced bias into the interview sample, meaning results echo the insights of a unique group of farmers and veterinarians with a certain relationship style or interaction quality linked to the south-west veterinary experience.

However, as the interview process involved the discussion of all experiences over the course of a participant's lifetime (exploring interactions with both current and past herd veterinarians or clients in addition to experiences with wider members of practice, advisory and on-farm teams), the effect of the current veterinarianfarmer relationship was felt to have been mitigated to a reasonable extent (all participants had both good and bad experiences to recount and reflect on given this broad focus). Additionally, while it is not possible to rule out a geographical influence, the prominence of relational factors in wider research on veterinarian advisory services (Richens et al., 2016) suggests that factors in this study are of broad relevance and not stringently bound to geographical divides. As a result, the authors feel these results can still offer meaningful insight to practicing veterinarians. As data saturation was reached, the opinions of this sample of farmers and veterinarians were also considered to be adequately evoked during the interview process.

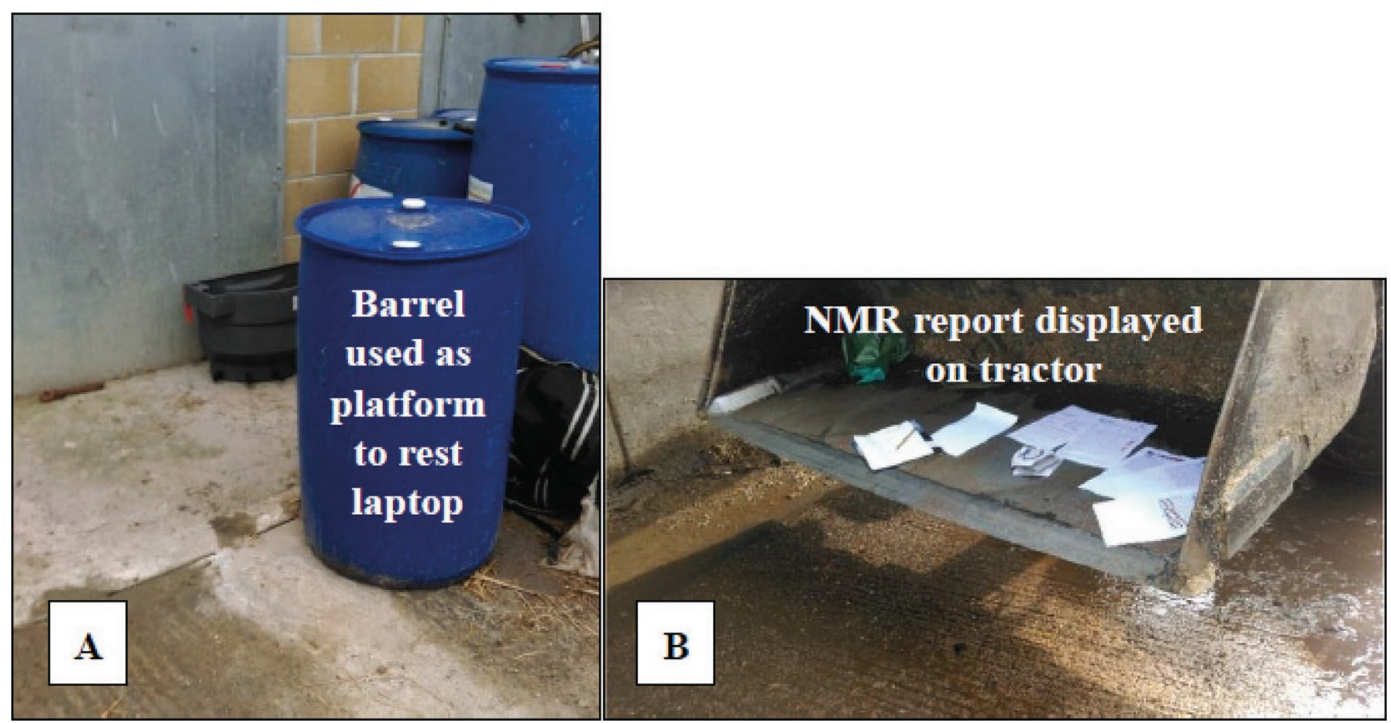

Figure 4. Examples of informal desks participant veterinarians used to integrate computer and paper-based reports into the dairy consultation paradigm. NMR $=$ National Milk Record. 
Integrating Themes: 3 Considerations for Building Engagement with Advice

When considering whether farmers are likely to engage with advisory recommendations, results suggest that veterinarians could benefit from considering not only the content and accuracy of their advice but also the local and relational context within which the advice is being transmitted. This could be achieved through attending to 3 core considerations suggested by the data:

\section{Consideration 1: Advice Must Manifest Meaning}

(a) Advice must align with the farmer's local world view, through resonating with the synergy of individual, social/cultural, and environmental influences that create such a world view (e.g., farmers' need to be "cautious on costs" while also being "a good farmer").

Competing personal influences create an internal narrative determining the interpretation and judgment of advisory recommendations; veterinarians should aim to evoke and understand this narrative in its complexity to target effective advice, rather than attribute advisory value to a single perceived factor (e.g., by assuming financial efficacy alone is (always) sufficient motivation for change). It is perhaps for this reason that veterinarian narratives and consultation paradigms intuitively reflect the need to develop and harness a shared understanding with the farmer to deliver recommendations with which farmers will engage.

If advice does not align with this world view:

(b) Advice must be of sufficient salience that this world view is reconfigured through relational attributes (e.g., becoming a practice specialist in an advisory area, forging a specific practice identity, or harnessing peer advisory support) and delivery attributes (e.g., utilizing mutualistic communication in advisory discussions, "showing the change" being advised in a practical and accessible manner).

These relational and delivery attributes that enhance advisory salience may in fact be embedded through the amplification of features identified as trustworthy virtues. For example, for relational attributes, if a speaker was recognized by a farmer as having special ability in a topic of interest, their recommendation for a specific change measure may resonate more strongly (for example, industry specialists). Similarly, when hearing a recommendation from another farmer, the virtue of integrity behind the advice may be amplified, where farmers report feeling peer messages on change reflect honest evaluation of an intervention: "they'll tell you the truth most of the time."

Similarly, for delivery attributes, aspects such as the tangibility of change, accessible delivery medium, and message consistency may embed integrity in advisory messages, given the sheer transparency of advisory efficacy. This perhaps contributes to the perceived effectiveness of benchmarking for engaging farmer motivation, as the sense of "seeing the change" in other farmers' practices is implicit in the process of data access and peer comparison, argued by Sumner et al. (2018) to stimulate instrumental value in the benchmarking process.

Communication attributes reported as desirable, those more akin to relationship-based approaches, may also embed greater feelings of advisor benevolence and integrity in advisory interactions, perhaps underpinning their association with enhanced client satisfaction (Coe, 2008) and enhanced adherence to veterinary recommendations (Kanji et al., 2012). It is possible that conscious and deliberate adoption of these features might therefore encourage advisory recommendations to manifest meaning for farm clients.

\section{Consideration 2: Promote Veterinary Trustworthiness}

Veterinary advisors must be considered to be in a place of trust, predicated on the trustworthy virtues of veterinarian ability, benevolence, integrity, and predictability; without this quality in the working relationship, advisory recommendations will not readily be integrated and enacted.

These components of trustworthiness set the virtuous stage for the advisory paradigm and give the information conveyed by the veterinarian meaning. For a trustworthy veterinarian, the farmer can reasonably assume that the advisory communication comes from someone with the appropriate knowledge, skill, and confidence to address the problem (ability); who will give care and consideration for the farmer's needs in deciding and advising on appropriate action (benevolence); is honest about the contextual benefits, drawbacks, and costs of this (or other) management choices (integrity); and whose continued support and insight can be relied upon when enacting the advice (predictability). If the legitimacy of one or more components is questionable, the decision to trust and use this trust to guide action would be expected to flounder (Dietz and Den Hartog, 2006); that is, a farmer's proclivity to accept vulner- 
ability and risk from the veterinarian's advice weakens and, with it, the resolve to enact advice:

Farmer 1: "Once you lost trust in a vet it's difficult. You start questioning everything. Probably $95 \%$ of his advice was absolutely spot on and wonderful, but a couple of things had led me to doubt him a little. I think once that's gone, it's no[t] good for anybody. I'd sooner start again with somebody else."

Indeed, this proclivity was recognized by Fisher (2013) who described trust as critical in building social capital between the farming community and external advisors, without which farmers' will lack confidence in the actions taken by these advisors and doubt the importance and usefulness of the recommendations they provide. Veterinarians considering why their farmers fail to listen and engage with their advice could consider this perception of trustworthiness as the first step in enactment of behavior. Careful consideration of how their farmer may perceive them across these trustworthy virtues may encourage them to alight on positive ways to enhance their interactions on farm.

\section{Consideration 3: Ensure A Perceived Shared Understanding Is Accurate}

The shared understanding between veterinarians and farmers reported by participants in this study is a critical contributor to successful target and delivery of advice; if the shared understanding between veterinarian and farmer is accurate, veterinarians will have a realistic understanding of the farmers' world view and thus whether an advisory recommendation will intuitively manifest meaning of require further attention to message saliency to build engagement. Indeed, in veterinarians' shorthand for farmer types (e.g., motivated/ unmotivated, proactive and reactive, and listeners and nonlisteners), veterinary participants already reported allowing this shared understanding to guide their recommendations with differing farm clients.

However, the reality of a shared direction within the herd health advisory paradigm is often elusive. Farmers and veterinarians differ in their opinions on what the veterinary advisor's main role is on farm (Hall and Wapenaar, 2012), and when polled, showed discrepancies in their prioritization of herd health topics (Derks et al., 2013). These discrepancies may in fact be underpinned by this very sense of shared direction and informality, for where veterinarians fail to make goals explicit with their clients, this is reported to in part be attributed to veterinarians feeling that (1) goal documentation is too formal, and (2) both veterinarians and farmers are aware of each other's wishes (Derks et al., 2013). Additionally, interview data suggest that the shared understanding may also mean communication on animal health topics is not always prioritized:

Veterinarian 7: "I like the long-term relationships with [clients]. I just sometimes wonder if because of that, we [don't] look at things as properly as we should do, because we always talk about other things, rather than cows."

As a result, this perceived consensus in herd health discussion creates 2 issues in the provision of animal health services. First, both parties are relying on their shared understanding to guide activity on farm, yet the consensus may to some extent be fictional; this consensus may be a perceptual product of a trusting relationship and embedded cultural script, rather than a measurable construct derived from mutual understanding of animal health priorities. Second, because of this perceived consensus, agenda setting within the clinical encounter does not demand substantive attention; if there is an implicit assumption of priorities under appraisal, it does not make sense to expend time (often perceived as valuable, limited, or costly in advisory interactions) on the tasks that typify agenda setting in the clinical encounter (Figure 5). This is to the detriment of the herd health consultation, as agenda setting offers numerous benefits within advisory encounters; in the medical sciences, both advisors and clients experience greater satisfaction with the clinical interaction given agenda setting processes, patients experience enhanced motivation toward positive behavior change for their illness and recovery, and time is more efficiently used (Gobat, 2014).

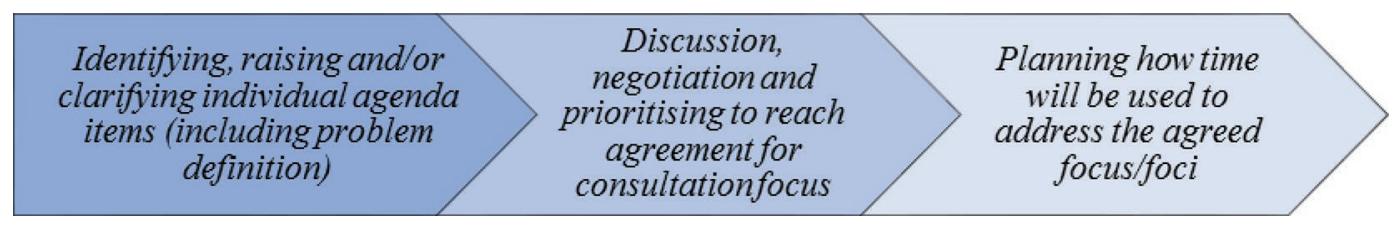

Figure 5. Agenda-setting tasks in the clinical encounter (Gobat et al., 2015). 
In lieu of these considerations, it is critical that the trusting and close working relationship so valued within this professional interaction is not conflated with an ability to accurately predict a farmer's immediate and long-term motivational drivers, which are complex and may vary temporally with evolving individual, social/ cultural, and environmental conditions. If the shared understanding between veterinarian and farmer is accurate, knowing whether an advisory recommendation will initially align with a farmer's world view or needs further attention to message saliency appears to be intuitive. However, given support for the assertion that this shared understanding is often mismatched (Derks et al., 2013), careful attention to communication about farmer goals and values should precede any such intuitive assumption on behalf of the veterinarian.

\section{A Practical Recommendation: Integrating Considerations in Practice}

Developing a collaborative consultation focus with farmer priorities, motivations, and goals recognized as paramount in framing and informing advisory messages could encourage veterinarians to deliver more appropriate, efficacious, and timely veterinary expertise through ensuring an accurate shared understanding of a farmer's world view. In turn, the farmer could be more likely to effectively integrate and enact recommendations, given their enhanced relevance for their unique personal and farming circumstances. This farmer-centered approach to veterinary interactions has the potential to establish a meaningful culture of change within the herd health advisory paradigm; active co-creation of plans between invested individuals stimulates better engagement and commitment in the tackling of complex problems (Steinlin and Jenkins, 2010).

Change-oriented, client-centered veterinary communication could support this need, with evidence-based methodologies such as motivational interviewing (Miller and Rollnick, 2012) encouraging "checking in" on the shared understanding ("Do I really know my farm client's goals and priorities for their farm right now?" and "Am I fully aware of what my farm client wants from this consultation?") and promoting effective engagement with advice ("Am I ensuring my farm client feels heard, respected, and autonomous in this discussion? What are their real thoughts on this change?") during the advisory interaction, while emphasizing virtues critical for trust (Bard, 2018). Education and training focused on veterinarians' clinical communication competencies is therefore well placed to support creating a culture of change within the herd health encounter, through refining interpersonal skills that attend to critical relational factors underpinning the enactment of veterinary advice.

\section{ACKNOWLEDGMENTS}

The authors wholeheartedly thank our funders the British Veterinary Association Animal Welfare Foundation (London, UK), The Co-operative Group (Manchester, UK), and Müller Milk and Ingredients (Market Drayton, UK) for assisting in recruitment and the anonymous farmers and veterinarians who gave their time and enthusiasm to support this research. We also thank our anonymous reviewers, whose comments helped us restructure and enhance this paper.

\section{REFERENCES}

Bard, A. 2018. Improving dairy cattle welfare: Examining Motivational Interviewing, veterinary communication and the herd health advisory paradigm. PhD Thesis, University of Bristol Veterinary School, Bristol, UK.

Braun, V., and V. Clarke. 2006. Using thematic analysis in psychology Using thematic analysis in psychology. Qual. Res. Psychol. 3:77-101. https://doi.org/10.1191/1478088706qp063oa.

Burton, R. J. F. 2004a. Reconceptualising the "behavioural approach" in agricultural studies: A socio-psychological perspective. J. Rural Stud. 20:359-371. https://doi.org/10.1016/j.jrurstud.2003.12.001.

Burton, R. J. F. 2004b. Seeing through the 'good farmer's' eyes: Towards developing an understanding of the social symbolic value of 'productivist' behaviour. Sociologia Ruralis 44:195-215.

Cannas da Silva, J., J. P. Noordhuizen, M. Vagneur, R. Bexiga, C. C. Gelfert, and W. Baumgartner. 2006. Veterinary dairy herd health management in Europe: Constraints and perspectives. Vet. Q. 28:23-32. https://doi.org/10.1080/01652176.2006.9695203.

Coe, J. B. 2008. Communication during veterinarian-client-patient interactions in companion animal practice. $\mathrm{PhD}$ Thesis, Department of Population Medicine, University of Guelph, Guelph, Ontario, Canada.

DEFRA. 2004. Animal health and welfare strategy for Great Britain. Accessed Apr. 8, 2018. https://www.gov.uk/government/uploads/ system/uploads/attachment_data/file/192951/animal-health -welfare-strategy.pdf.

Derks, M., B. van Woudenbergh, M. Boender, W. Kremer, T. van Werven, and H. Hogeveen. 2013. Veterinarian awareness of farmer goals and attitudes to herd health management in The Netherlands. Vet. J. 198:224-228. https://doi.org/10.1016/j.tvjl.2013.07 .018 .

DiCicco-Bloom, B., and B. F. Crabtree. 2006. The qualitative research interview. Med. Educ. 40:314-321. https://doi.org/10.1111/j.1365 -2929.2006.02418.x.

Dietz, G., and D. N. Den Hartog. 2006. Measuring trust inside organisations. Person. Rev. https://doi.org/10.1108/00483480610682299.

FAWC. 2011. Education, Communication and Knowledge Application in Relation to Farm Animal Welfare, (December), pp. 1-37. Accessed Apr. 7, 2018. https://www.gov.uk/government/uploads/ system/uploads/attachment_data/file/324908/FAWC_report _on_education_communication_and_knowledge_application_in _relation_to_farm_animal_welfare.pdf.

Fisher, R. 2013. "A gentleman's handshake": The role of social capital and trust in transforming information into usable knowledge. J. Rural Studies 31:13-22. https://doi.org/10.1016/j.jrurstud.2013 .02 .006 .

Gobat, N., P. Kinnersly, J. W. Gregory, and M. Robling. 2015. What is agenda setting in the clinical encounter? Consensus from literature review and expert consultation. Patient Educ. Couns. 98:822-829. 
Gobat, N. H. 2014. Agenda setting in the clinical encounter: What is it, and is it measureable? PhD Thesis, School of Medicine, Cardiff University, Cardiff, UK.

Hall, J., and W. Wapenaar. 2012. Opinions and practices of veterinarians and dairy farmers towards herd health management in the UK. Vet. Rec. 170:441. https://doi.org/10.1136/vr.100318.

Jansen, J. 2010. Mastitis and farmer mindset. Towards effective communication strategies to improve udder health management on Dutch Dairy farms. Wageningen University, Wageningen, the Netherlands.

Kanji, N., J. B. Coe, C. L. Adams, and J. R. Shaw. 2012. Effect of veterinarian-client-patient interactions on client adherence to dentistry and surgery recommendations in companion-animal practice. J. Am. Vet. Med. Assoc. 240:427-436. https://doi.org/ 10.2460/javma.240.4.427

King, N. 2004. Using templates in the thematic analysis of text. Pages 256-270 in Essential Guide to Qualitative Research Methods in Organisational Research. SAGE Publications, Newbury Park, CA.

Mee, J. F. 2007. The role of the veterinarian in bovine fertility management on modern dairy farms. Theriogenology 68(Suppl. 1):257265. https://doi.org/10.1016/j.theriogenology.2007.04.030.

Miller, W. R., and S. Rollnick. 2012. Motivational Interviewing: Helping People Change. 3rd ed. Guilford Press, New York, NY.

NMR. 2018. Introduction to NMR. Accessed Apr. 7, 2018. https:// www.nmr.co.uk/about.

QSR International. 2018. What is NVivo? NVivo. Accessed Apr. 8, 2018. http://www.qsrinternational.com/nvivo/what-is-nvivo.

RCVS. 2018a. Postgraduate Qualifications: Certificate in Advanced Veterinary Practice (CertAVP), Lifelong learning. Accessed Apr. 8, 2018. https://www.rcvs.org.uk/lifelong-learning/postgraduate -qualifications/certificate-in-advanced-veterinary-practice -certavp/.

RCVS. 2018b. Professional Accreditation: Advanced Practitioner status, Lifelong learning. Accessed Apr. 8, 2018. https://www.rcvs .org.uk/lifelong-learning/professional-accreditation/advanced -practitioner-status/.

Richens, I. F., P. Hobson-West, M. L. Brennan, Z. Hood, J. Kaler, M. Green, N. Wright, and W. Wapenaar. 2016. Factors influencing veterinary surgeons' decision-making about dairy cattle vaccination. Vet. Rec. 179. https://doi.org/10.1136/vr.103822.

Ritter, C., J. Jansen, S. Roche, D. F. Kelton, C. L. Adams, K. Orsel, R. J. Erskine, G. Benedictus, T. J. G. M. Lam, and H. W. Barkema. 2017. Invited review: Determinants of farmers' adoption of management-based strategies for infectious disease prevention and control. J. Dairy Sci. 100:3329-3347. https://doi.org/10.3168/ jds.2016-11977.

Roter, D. 2000. The enduring and evolving nature of the patient-physician relationship. Patient Educ. Couns. 39:5-15. https://doi.org/ 10.1016/S0738-3991(99)00086-5.

Ruston, A., O. Shortall, M. Green, M. Brennan, W. Wapenaar, and J. Kaler. 2016. Challenges facing the farm animal veterinary profession in England: A qualitative study of veterinarians' perceptions and responses. Prev. Vet. Med. 127:84-93. https://doi.org/ 10.1016/j.prevetmed.2016.03.008

Steinlin, M., and C. W. Jenkins. 2010. Knowledge Sharing for Change: Designing and Facilitating Learning Processes with a Transformational Impact. Ingenious Peoples Knowledge Consultants, Cape Town, South Africa.

Sumner, C. L., M. A. G. von Keyserlingk, and D. M. Weary. 2018. How benchmarking motivates farmers to improve dairy calf management. J. Dairy Sci. 101:3323-3333. https://doi.org/10.3168/jds $.2017-13596$

Vaarst, M., T. B. Nissen, S. Østergaard, I. C. Klaas, T. W. Bennedsgaard, and J. Christensen. 2007. Danish stable schools for experiential common learning in groups of organic dairy farmers. J. Dairy Sci. 90:2543-2554. https://doi.org/10.3168/jds.2006-607.

Vanclay, F., and G. Enticott. 2011. The role and functioning of cultural scripts in farming and agriculture. Sociol. Ruralis 51:256-271.

\section{APPENDIX}

Table A1. Veterinarian communication behaviors, attributes, and ethos reported as desirable or undesirable by farmers, veterinarians, or both

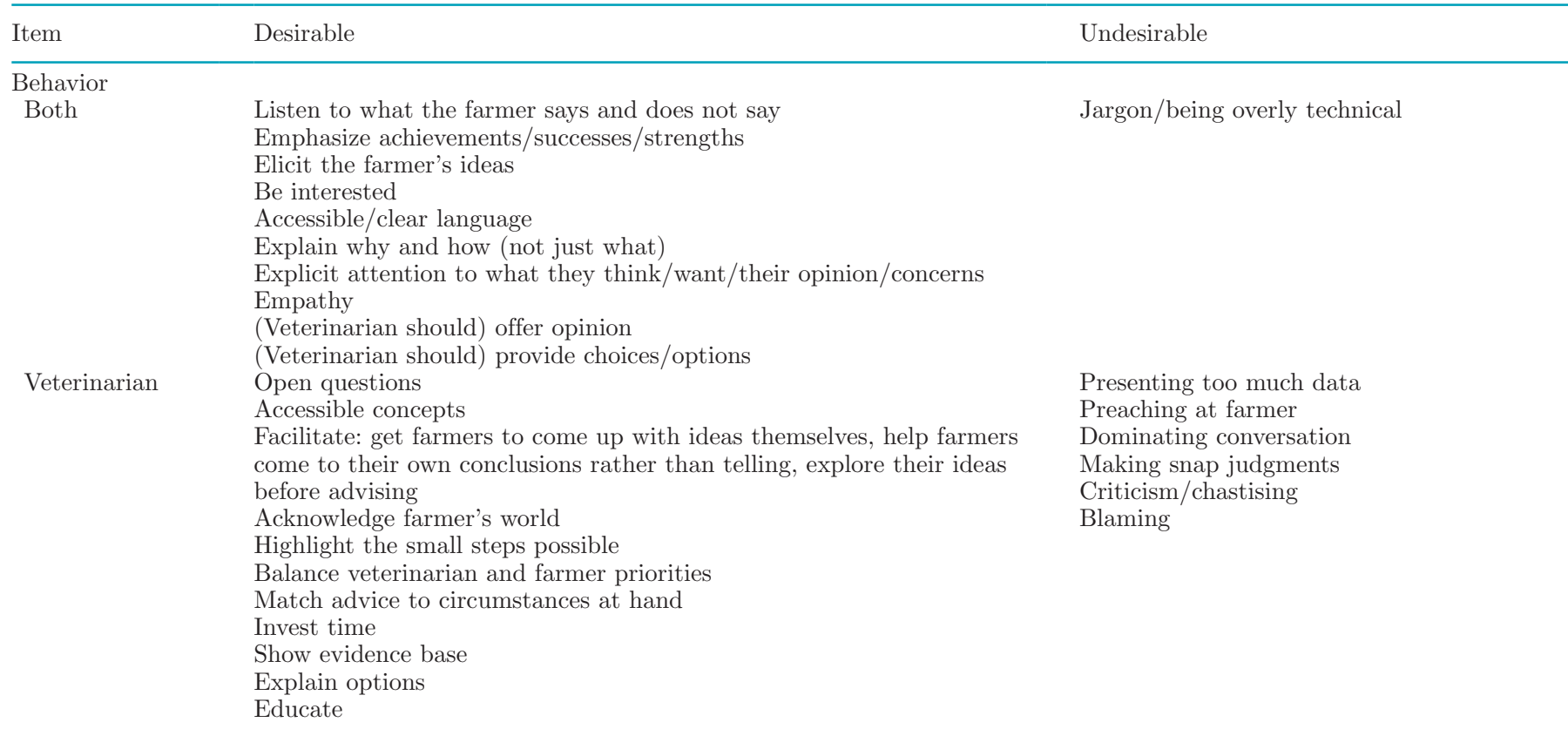


Table A1 (Continued). Veterinarian communication behaviors, attributes, and ethos reported as desirable or undesirable by farmers, veterinarians, or both

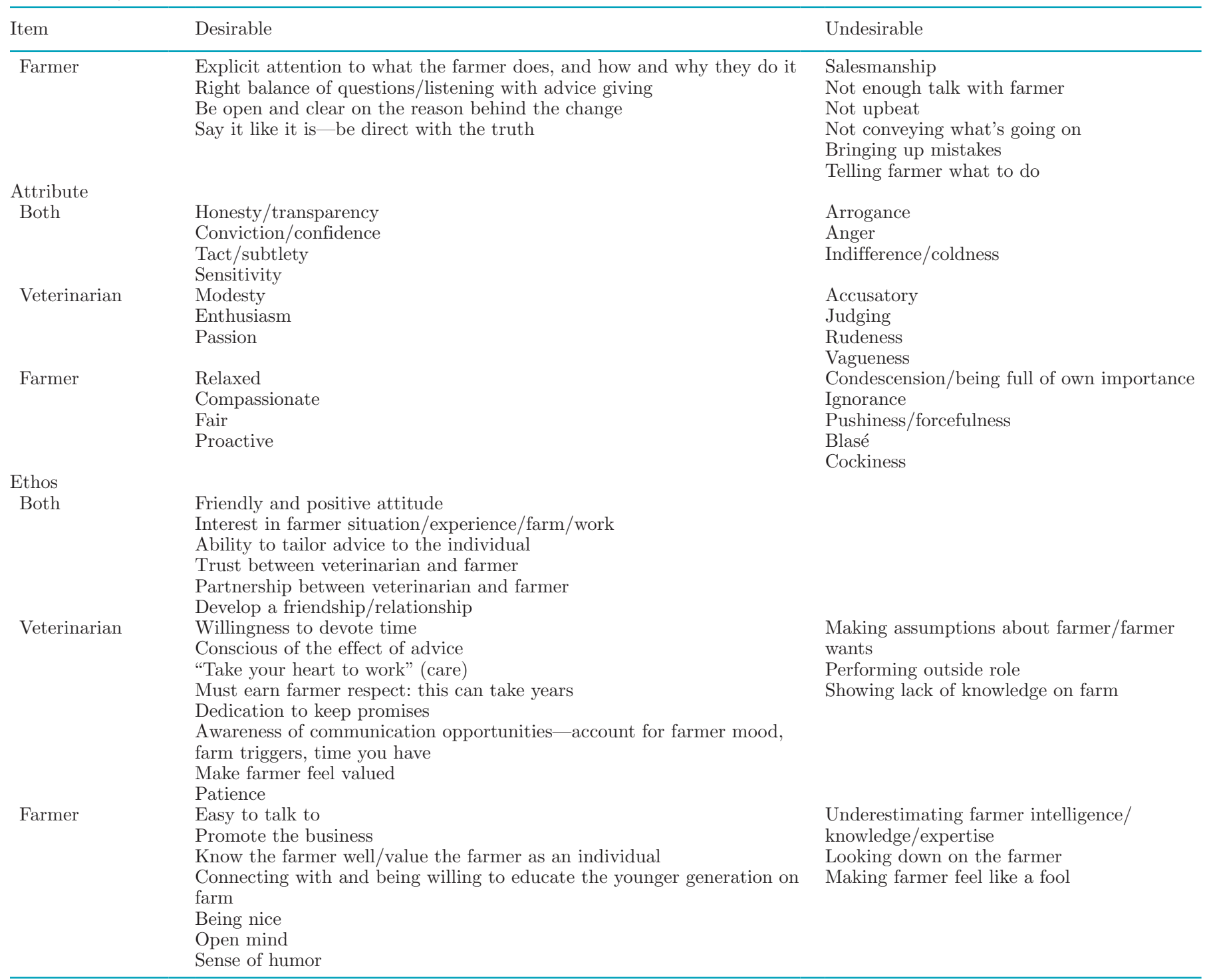

